\title{
Space presents and body experience: LouYe film's city imagination Liu Yunfei
}

Chongqing University of Posts and Telecommunications, Nanan district, Chongqing, china livyunfei@163.com

Keywords: LouYe, City film, Space, Body.

\begin{abstract}
Contrasts to the villages, cities in Chinese movies are not fully expressed. Since the $90 \mathrm{~s}$, the sixth generation has become the main directors for city films. It is different from other groups that they based on urban/reality of cultural psychology and express viewpoint, presented the cities they have seen. To Understand LouYe's city film, we need to begin from space and body on which he reflects city emotional experience, make us see the person's psychology landscape behind city landscape. It is a kind of self expression under the perspective of city.
\end{abstract}

\section{娄烨电影的城市想象：空间呈现与身体体验 刘云飞}

重庆邮电大学传媒艺术学院, 南岸区, 重庆, 中国 livyunfei@163.com

关键词: 娄烨; 城市电影; 空间; 身体

中文摘要. 对比乡村, 城市在中国电影里的表达并不充分。90年代以来, 第六代导演成为城 市电影的主要言说者, 与其他导演群体不同, 他们立足于都市/现实的文化心理及表达视点, 呈现了他们眼里的城市。理解娄烨的城市电影, 需要从空间与身体出发。他以此来观照都市 人的情感体验, 让我们看到都市繁华景观背后人的心理景观, 是一种自我视角下的城市表达。

\section{1. 引言}

对比乡村，城市作为电影的萌芽、扎根、繁荣之地，在中国电影里的表达并不充分。建 国以来，中国电影里的城市或等同于乡土的概念，或等同于 “都市里的村庄”，大部分导演 试图从道德评价、政治批判、历史反思的角度勾勒出文明与愚昧、现代与落后的图景。伴随 着现代化城市的进程, 怎样表现城市空间、城市体验, 以此来跟进城与人的现代性, 无疑成 为众多导演的思考题。80年代以来, 真正具有现代都市意识并带来城市讨论话题的电影始于 张泽鸣的《太阳雨》和孙周的《给咖啡加点糖》, 二者试图从文化心理的角度切入都市人面 对现代化都市理性上肯定/感情上否定的双重矛盾心态。此后，第六代导演携带着城市标记， 以带有自传性的生活经验为表现内容, 开始了第六代人的城市书写。娄烨是第六代群体中特 立独行的一个, 从《周末情人》开始, 他执着于都市空间和都市人的情感心理的表达, 既不 同于师辈, 又区别于同辈, 因此, 将娄烨作为一个个体来考察, 将具有重要意义。本文首先 厘清了娄华所属群体的文化心理及表达视点: 都市/现实, 并选择了两个解读的视角: 空间与 身体, 从而得出他对城市的一种想象化表达。 


\section{2. 乡土/历史还是都市/现实}

80 年代中后期以来，不同代际的导演群体均有涉及城市电影，但立足点不同。理解娄烨 的城市电影, 我们需要首先厘清他所属群体的文化心理及表达视点。长期以来, 乡土与都市 的概念在中国的电影文本里呈现互文性的特点, 理解城市电影需要借助乡土电影作参照, 汪 晖的《当代电影中的乡土与都市: 寻找历史的解释与生命的归宿》 ${ }^{[1]}$ 认为, 第五代导演以 “精 神流放者” 的心态来看待那片 “黄土地”，他们身上带着城市文化的印痕， “面对着都市文 明的混乱、非道德化和精神的颓丧, 这批 “精神流放者” 一方面身陷其中, 不由自主, 另一 方面又产生了深刻的叛逆与疏离的情怀”，他们一方面批判乡土礼俗的愚昧、落后，另一方 面又为黄土地所蕴含的原始的、神秘的力量所深深吸引, 以此来寻找寄托。同现代化城市共 同成长的第六代导演面对城市，与第五代导演面对乡土的矛盾心态基本相似，但他们并无 “流 放者” 的经历, 因此他们的困惑无法延伸到城市以外的那片 “黄土地” ，不管以何种方式抗 拒城市, 困扰依然存在, 依然停留在城市。立足当下的表述视点, 并没有解决娄烨等人的都 市困扰, 反而让他们走入了更深的陷阱。那么如何来解决他们的都市困恼? 王海洲的观点可 以给我们一点提示, 他在《视点及其文化意义: 当代中国城市电影研究》一文中认为, 90年 代以来的中国城市电影呈现出 “反城市化” 的特点, 从不同创作群体来看, 这种 “反城市化” 主要是从两个视点来切入的, “一个是第五代导演肩负历史使命的宏大叙事视点; 一个是先 锋导演的个体抗争式边缘视点” [2] 以第六代为代表的先锋导演, 以高傲的姿态来抗拒城市的 “拜物狂潮” , 试图保持自我的精神洁癖, 但同时又难敌都市物欲的侵染, 以放纵自我的方 式投入到都市快感的寻找之中，在物质和精神的撕扯下，他们最终选择了精神，但这并没有 解决他们的都市困扰, 这种精神自虐反而导致了他们情感心理的分裂。没有了土地的牵绊, 他们只能执着于青春迷恋, 以消耗精神和消费身体的方式抗拒城市, 试图为自己的困惑寻找 一个答案, 但这个答案却是无果的。

对比乡土/历史的表达视点, 我们可以发现未经 “精神流放” 的第六代群体的城市表达是 立足于都市/现实的, 并且指向两个层面: 空间与身体。我们可以从以下三个方面来证实: 一 是以现代城市表象为空间构建的立足点, 以街道、酒吧、舞厅、游戏厅、咖啡馆、电影院等 具有流动感的和城市标志性的景观为主, 没有了一望无际的黄土地, 没有了具有乡土寓意的 胡同与四合院; 二是以城市边缘人群为表述对象, 如摇滚青年、民工、落魄的艺术家、同性 恋、妓女、大学生等是; 三是试图扫描都市人的内心体验, 面对全球化与现代城市化进程, 他们内心深处经历着激烈的震荡, 与城市若即若离的关系恰如他们内心的孤独、边缘。对于 一向执着于自我电影理念的娄烨来说, 这种坚持更为彻底。作为第六代群体的一员, 娄烨的 城市表达同样立足当下, 着眼于反抗的姿态, 但并不是以 “我的摄影机不撒谎” 式的客观实 录, 而是有意选择湿漉漉、灰蒙蒙的城市景观, 以此来映祄当代都市人的内在世界, 并有意 编织人与时代、人与历史、人与环境、人与人、人与自我的复杂关系, 试图为困惑的情感世 界寻一个出路。娄烨的城市表达重在呈现, 而不在于评判, 重在一个问题的提出, 并不在答 案的给予。

\section{3. 公共空间与私密空间}

在论述城市电影时, 城市空间是一个不能回避的话题。不同的城市表象构成了不同的城 市形态，而不同的城市形态则表征着不同的城市文化内涵。娄烨电影的城市空间不同于 “由 胡同、四合院组成的 “都市里的村庄” ” [3], 也罕有高楼大厦、商业街、立交桥等现代建筑 的展示, 而主要是对以街道延展而来的酒吧、舞厅、游戏厅、咖啡馆、电影院等公共空间以 及以旅馆、家庭为主的私密空间的展现。选择什么样的表象来标记城市, 意味着赋予了城市 何种特征。刘海波、祁媛的《表述上海的三种方式: 三部城市传记与三种历史观》认为: “城 市是有性别的, ” ${ }^{[4]}$ 。比如山东与粗狂豪饮的山东大汉的对应, 苏州与吴侬软语的评弹女子 
的对应，而以成功表述女性形象为主的上海则呈现出 “女性化” 的特征。娄烨拍摄了关于多 个城市的电影，如北京、上海、南京、武汉、重庆、巴黎等，虽然其城市电影常常涉及女性、 同性恋等，但并未呈现出 “女性化” 的特点，也非 “男性化”，而是人物气质与城市气质的 契合，如导演所言： “城市选择基本上根据故事、剧本，然后看人物适合在哪一种环境里头， 每个城市就跟人一样, 它有它的特点。” 可以肯定地说, 娄烨电影中的城市无法用性别来界 定，但可以用片中人物的内心维度来评说。

\section{1 公共空间暗示着人物心理}

娄烨的城市电影很少表现现代都市繁华、时尚、鲜亮的一面，多表现城市黑暗、潮湿、 衰败的一面。充满商业气息和时尚气息的商业街和商务区消隐在背景处，凸显在前景的则是 充满流动性和暧昧性的以街道为主并延展而来的舞厅、酒吧、游戏厅、咖啡馆、电影院等。 即使是取材于 “旧上海” 的《紫蝴蝶》（2003年）也并未浮现旧上海的鬼影，没有强调穿旗 袍的女人和麦克风飘出的酒吧小调、舞厅浪歌。而取材于 “新上海” 的《周末情人》（1993 年）、《危情少女》（1995年）、《苏州河》（2000年），也没有上海其繁华的一面。无论 新旧, 娄烨都有消解上海这个城市在观众体验中一贯的梦幻性, 而立足于当下视点来建构过 去, 或再现现在。在其影片中, 摄像机以一种现在进行时的长镜头的跟进方式, 跟着人物在 街道上漫游, 跟随人物出入古旧住宅, 在舞厅、酒吧、游戏厅、咖啡馆、电影院里进进出出。 如在 《周末情人》开篇即是摄像机跟随阿西穿越狭窄的楼梯和灰暗的通道，去寻找他的情人 李欣; 《苏州河》开篇，摄像机沿着苏州河沿岸快速扫描衰败的码头、破旧的住宅、来来往 往的船只, 还有那些眼神呆滞的渔人、工人。

以街道为代表的公共空间表层指涉着都市灰暗、边缘、混乱、噪杂、落后的生存环境, 这里充斥着绑架、凶杀、政治暗杀、强奸等犯罪行为，深层指涉着都市流动性、暧昧性、不 确定性的一面。娄烨电影的人物在不同的空间里自由活动着，以游戏的态度寻找发泄情感和 青春愤怒的方式，但这些公共活动空间并未让他们建立与环境的情感连结，并未建立自我的 身份认同，反而离主流社会越来越远，遭遇了更大的孤独和不安。《春风沉醉的夜晚》（2009 年）中，同性恋江城在夜幕降临之时频繁出入同性恋酒吧，以易装（坦胸露背、烈焰红唇） 的方式在舞台上华丽高歌，但表演之后，却常常是他一个人蜷缩着哭泣的场景。

此外，娄烨在表现城市的公共空间时特别喜欢用水的意象，其影片有大量的情节是在夜 晚、雨天或是雨夜中进行的, 雨的潮湿、夜的黑暗、雨夜的凌乱, 加上环境空间的阴暗狭小, 使其影片具有凌厉的现实感。雨的使用一方面象征着一种焦虑、烦躁、压抑的氛围，让整个 城市变得湿漉漉、灰蒙蒙, 变得阴郁和灰暗, 另一方面也象征着人物平静情感海面下的暗潮 涌动。片中人物在经历重大的生活转折或情绪波折之后, 在心情异常低落之时, 他们多会在 雨夜中穿行，行走或奔跑。《浮城谜事》（2012年）中有大量雨中段落，密集的雨将环境的 压抑渲染到极致, 也将人内心的狂乱泄露, 正是在雨的掩饰下, 陆洁和桑琪杀死了 “小四” 蚊子, 乔永照杀死了勒索他们的流浪汉。

\section{2 私密空间揭示了人物关系}

娄烨的城市电影中有大量空间段落是关于旅馆和 “家庭” 的, 这里的旅馆等同于 “开房” 的含义, 而 “家庭” 并不是家族的概念, 也不是指由一夫一妻构成的单元, 而是指人物的活 动场所。颇能体现现代化城市进程的旅馆和 “家庭” 并未显现它们应有的一面, 也并不是幸 福、和谐、温馨与舒适的指代。摄影机很少对旅馆和 “家庭” 空间作全景扫描, 而是使用他 惯常的手摇镜方法, 镜头快速地从房间里一个人的中近景摇至另一个人的中近景, 镜头里没 有高档小区的标志, 没有现代化设施的展现, 镜头常常聚焦房间的一隅, 比如卫生间、卧室、 一张床等等。这个房间的环境如何并不重要, 重要的是房间里的人以及人与人之间的关系。 如果说以街道为代表的都市空间具有开放性和流动性的特点, 那么以旅馆为代表的都市空间 则具有封闭性和私密性的特点。 
私密空间将外部的宏观世界缩小为内部的微观世界, 是导演投射主体想象的舞台, 它的 封闭和狭窄使其充满戏剧性, 也提供了一种新的审视人物关系的视角, 让观众来看人物之间 亲疏的变化。影片《花》（2011年）接近一半的场景是在旅馆和家（人物暂时租住的房间） 的室内拍摄的, 花与马修由陌生到亲密, 由亲密到疏远的变化过程都是在室内完成的; 《春 风沉醉的夜晚》江城与王平、罗海涛的关系也是在旅馆和家中开始与结束的, 影片结尾闪回 至江城和王平依偎在一起, 朗读郁达夫的《春风沉醉的晚上》的段落, 人物的对白和镜头由 室内到室外的摇出, 让我们及时抽离故事营造的情景, 判断人物之间的关系。两部影片里人 物关系的变化充满戏剧性, 而这种戏剧性在狭窄、灰暗的室内空间里得到强化。影片里人物 均以身体对话来取代言语沟通, 虽然更赤裸但却更直接、更彻底。《浮城谜事》难得出现一 个相对完整的家庭, 影片开篇既是男主角乔永照出差回来与妻子、女儿在家庭里团聚的欢乐 画面, 但这个画面随后被乔永照出现在另一个有自己妻子与儿子的家庭画面所解构。实际上, 乔永照表面上有一个幸福的家，背后还有另一个藏在暗处的家，还有发生一夜情的女朋友。 最后这种关系在两个家庭的 “大团圆” 中被撕裂：陆洁与女儿安安在桑琪与宇航家里（乔永 照的另一个妻子与儿子), 这时乔永照推门进来, 明暗两个家就这样见面了。娄烨将生活的 悬念和戏剧性浓缩在一个有着双城性质的家庭里, 可以说, 正是在家这样一个特殊的空间里, 人物之间的关系才有了爆发的张力。

\section{4. 身体体验：爱情/性}

从20世纪后期开始, 无论还是社会学科还是人文学科都不由自主地表现出对身体研究的 兴趣。正如尼采呼喊着 “一切从身体出发”, 身体开始从意志的钳制下被解放出来。身体的 觉醒促进了现代视觉消费文化的产生, 而大众传媒又反正来规训着内在与外在的身体, 关于 身体维护与保养的视觉符号充斥都市空间, 成为都市文化与都市形象的重要组成部分。而在 中国电影史中, 自新中国成立以来, 身体问题长期被忽略与被遗忘, 身体暴露与性展示更被 视为禁忌, 或者身体被附着了政治/社会的符码, “尤其是女性身体受伤的视觉主题, 常常成 为国家民族灾难的历史隐喻，从而超越身体本身所指，带上了社会文化符号的特性”。 ${ }^{[5]}$ 在 八九十年代以来的中国银幕上, 身体表现有了突破性的进展, 《红高粱》、《菊豆》、《良 家妇女》等乡土影片开始凸显被压抑的身体, 而娄烨的城市电影则将身体展示放在了都市空 间中, 在视觉文化和消费文化的背景下, 通过对身体体验: 爱情/性的呈现, 试图建立人物自 我意识与身体的认同机制, 再现当代城市生活空间。爱情/性是身体欲望的重要显现, 爱情与 性又不可剥离。在娄烨看来: “性或者爱情看起来是很隐私, 甚至不大的事情, 特别局部东 西，但是从它开始，可以延伸到很多问题，看到人性的东西，看到社会背景的东西。” [6] 他的电影里或多或少都涉及大尺度的性爱描写, 这是人物身份焦虑的体现, 也是他们人性的 一部分, 他们以此来感知自我存在, 如娄华所说： “性是自由的和自然的人的不可缺少的一 个方面”。

一方面, 他以爱情作为读解人生、阐释世界的视角, 并带出广阔的城市生活图景。娄烨 反复说到: “爱情它其实不只是爱情, 还涉及很多其它方面”, “这是一个最丰富的视角” , “关注微观层面上的东西——比如爱情, 就能更接近宏观的本质。” [7]《周末情人》中的爱 情被放在了以摇滚乐、地铁、可口可乐、万宝路香烟等为标志的 “上海世界主义” 的图景当 中, 刚刚走上改革开放之路的上海, 已表现出某些物欲化的倾向, 片中的人物在这样的环境 里漫游, 满腔的愤怒无处发泄, 只能以爱情来寻找安慰, 他们的爱情与其说是爱情, 不如说 是青春愤怒的冲动。《紫蝴蝶》以爱情牵扯出多重人物关系, 丁慧与伊丹、丁慧与谢明、丁 慧与司徒、司徒与伊玲等, 以谢明和丁慧为代表的地下间谍组织, 带着对死亡的恐惧和战栗, 盲目冲动地投入暗示的战斗, 并导致无辜的伊玲当场皆命, 让司徒阴差阳错地卷入一场谋杀 之中, 从此走上了复仇的不归路。影片让摄影机始终处在平视的视角讲述了一段 30 年代的上 海故事, 即以当下视角来反观历史。30年代的时代洪流对人物命运的携裹恰似当今现代化城 
市对人的冲击，生命本无可保证，而附着的爱情又从何言起？ 《苏州河》在肮脏的苏州河沿 岸为我们解构并建构了一个爱情童话, 马达以执着的寻找向牡丹证明了爱情的存在, 却同时 让美美（另一个牡丹）开始质疑爱情，通过拥有一质疑一追寻的过程来反映现代化城市进程 所扫荡的个人记忆。《濒和园》的女主角于虹把爱情当作生命来追求, 在不同的时空下尽情 坦露着爱情的欲望, 并由此带出跨越十五年和六个城市的广阔时空, 具有强烈的时代况味和 怀旧色彩。被禁期间拍摄地《春风沉醉的夜晚》、《花》同样关于爱情, 一个加入了同性恋 的元素, 一个加入了异国漂泊的背景, 并以大尺度的性爱描写来表现特殊环境下人物茫然、 焦灼的精神状态。

另一方面, 在不同的生活时空下, 娄烨电影中的爱情故事呈现出不同的面貌, 并以此来 探讨都市人的都市情绪——情感的困乏与渴求。“爱情首先是一种身体的感受, 它是产生在 逻辑, 思想, 概念, 道德等等之前的一种感受, 所以它也是离人的最本身的东西最近的, 所 以它人性，美好，又危险。”[8]性爱如放大镜般把都市人内心荒漠般的情感世界放大呈现。 如果说《周末情人》中的爱情更像是青春期的冲动, 《苏州河》中娄烨让我们相信爱情童话 的存在, 《臨和园》中的于虹把爱情当作生命, 《春风沉醉的夜晚》让人开始质疑爱情的存 在, 同性恋看似是爱情与婚姻的终结者, 其实仅仅是爱情幻灭、婚姻破裂的诱因。同性恋的 出现只是加速了人性本质暴露的速度, 让人更快速更清楚地看清了内心的欲望、洞悉了现在 爱情的易变和脆弱, 彻悟了真爱的难寻与坚守的艰难。《花》的留学生花把爱情等同于性, 性的开始就代表着爱情的开始, 性的结束也注定爱情即将被放弃。而《浮城谜事》则将关于 幸福美满的婚姻爱情之梦彻底粉碎。片中的乔永照打着爱的旗号一次又一次的猎艳, 在一个 个年轻的身体上发泄着、探寻着, 他似乎不为性而性, 不为爱而爱, 就这样迷失在城市的浮 华与喧器之中。他拥有当代城市生活中奢侈的一切: 和谐的家庭, 幸福的婚姻, 成功的事业, 但正是这些表面的富足导致了他情感和心理的畸形和分裂, 他隐藏着身份奔走在两个家庭之 间, 他时而暴力又时而温情, 我们不知道他缺失的到底是什么, 要寻找的到底是什么, 他所 认同的到底是什么? 少女蚊子的惨死暴露了人性的复杂, 也让生活的真相、生命的真实变得 更加扑朔迷离。从上述影片的分析, 我们可以看出, 随着时间的推进, 娄烨影片的人物对待 性爱的态度有一个变化的过程, 从追寻到相信到质疑再到破坏, 我们无法猜测这是否就是导 演自身爱情观的一个变化过程, 但我们可以说这反映了人物对现代城市生存状况的一个态度、 情感的变化，反映了都市人情感、心态的多元化，也反映了导演探讨层次的深入。

\section{5. 结束语}

无论是城市空间的建构还是性爱的呈现，娄烨都有意将镜头转入人的内心世界，立足于 当下的感受与体验, 通过情欲冲突以及人们对性的态度, 反映城市环境对人物情感世界的影 响。娄烨让我们看到了现代繁华都市背后的都市人的心理景观, 在一部分人哪里, 情感的丰 饶与物质的充裕并不成正比, 总有一部分人孤独而不安, 在喧华的都市街景中寻寻受受, 无 处安放自己的魂灵。这同样在导演拍电影的方式中得到体现。仔细考察娄烨的作品, 我们可 以发现摄影机始终处于平视的视角来观察世界, 以人物的中近景、脸部的特写为主, 他很少 使用从高处俯拍的全景镜头, 虽然有很多城市环境的段落是以跟拍、隐藏镜头、街道流动作 战完成的, 但是通过手摇镜头、变焦镜头、快速运动的处理, 焦点始终在人物的脸上, 现实 环境很难以真实的姿态显现, 世界仿佛都隐到了人物的后面, 成了虚化的背景。娄烨所关注 的并不是历史事件本身或社会发展变动, 而是生活在其中的人, 通过人的体验、挣扎、逃离、 回归来看人对生存世界的反应及态度。因此, 我们可以说他的城市是他操作摄影机的舞台, 带有很明显的个人化的印痕，是关于一座城市的想象，是一座想象的城市。 


\section{致谢}

本文为2017年度教育部人文社会科学研究青年基金项目（项目批准号：17YJC760055)及 2016年度重庆市社会科学规划项目（项目批准号：2016BS028）的阶段性成果之一。

\section{References}

[1] Wang Hui. The local and urban in contemporary film: to find the interpretation of history and the fate of life [J]. Film Art, 1989, (3).

[2] Wang Haizhou. Viewpoints and their cultural significance: contemporary Chinese city film research [J]. Film Art, 2005, (3).

[3] Chen Xiaoyun. Film City: Chinese film and city culture (1990-2007) [M]. Beijing: China Film Publishing House, 2008:29.

[4] Liu Haibo, Qi Yuan. Three ways to express Shanghai: three city biography and three historical views [J]. Contemporary film, 2012, (10).

[5] Chen Xiaoyun.Characteristic significance of physical trauma in Chinese cinema [J]. Literature and Art Research, 2008 (3).

[6] Lou Ye: during the prohibition period I was a hermit. Http: //ent.qq.com.

[7] Liu Min, Lou Jun. Best Screenplay Award: Mei Feng ("Spring intoxicated night") Lou Ye: do your own master [J]. The film world, 2009 (6).

[8] Zhao Jing. Static distance observation of the three: Liu Jie, the woman who fell in love with Lou ye.Http://ent.sina.com.cn. 